\title{
Fine-Grained Prediction of Cognitive Workload in a Modern Working Environment by Utilizing Short-Term Physiological Parameters
}

\author{
Timm Hörmann, Marc Hesse, Peter Christ, Michael Adams, Christian Menßen and Ulrich Rückert \\ Cognitronics and Sensor Systems Group, CITEC, Bielefeld University, Bielefeld, Germany \\ thoerman@techfak.uni-bielefeld.de
}

\begin{abstract}
Keywords: $\quad$ Fine-Grained, Cognitive Workload, Stress, Heart Rate, Electrodermal Activity, Tablet Computer, Human Machine Interaction, Industry 4.0

Abstract: $\quad$ In this paper we present a method to predict cognitive workload during the interaction with a tablet computer. To set up a predictor that estimates the reflected self-reported cognitive workload we analyzed the information gain of heart rate, electrodermal activity and user input (touch) based features. From the derived optimal feature set we present a Gaussian Process based learner that enables fine-grained and short term detection of cognitive workload. Average inter-subject accuracy in 10-fold cross validation is $74.1 \%$ for the fine-grained 5-class problem and $96.0 \%$ for the binary class problem.
\end{abstract}

\section{INTRODUCTION}

Today's modern working environments are increasingly challenging for the employees. As one example the concept of "Industry 4.0" sketches the design of new flexible working environments, in which employees are constantly confronted with new requirements. This implies manufacturing processes with very small lot sizes, which will result in a higher diversity of working processes. It forces the employees to be highly flexible and to adapt rapidly to changing work tasks. For instance, the employees will have to memorize and apply new knowledge more often (Botthof and Hartmann, 2015). Therefore, adaptive HumanMachine-Interaction (HMI) becomes more important. Especially by means of the implementation and utilization of adaptive assistive systems, which shall be used to guide an employee through a new and unfamiliar task (Wallhoff et al., 2007). With the ability to balance the cognitive workload (CW) of a specific task, the ergonomic design of working tasks could be improved. This makes the prediction of $\mathrm{CW}$ a key factor towards human centric design, concerning the development of adaptive HMI and adaptive assistant systems. (Rouse et al., 1993) (Wallhoff et al., 2007)

To fulfill the requirement of adaptability, an assistive system needs to know the human users' cognitive capacity. Therefore, in order to adjust correspondingly to the user's needs, it is important to precisely model the user's perceived CW. The goal is to balance the complexity of a given task. This is be- cause, on the one hand, if the user is not sufficiently assisted, it might lead to mistakes. But on the other hand, if the user feels unchallenged, it might decrease his attention (Young and Stanton, 2002). Because both lead to frustration, the prediction of $\mathrm{CW}$ has to be as precisely and therefore as fine-grained as possible. To prevent such situations, an adaptive assistive system, as an example, could increase or decrease the amount of supporting information provided or the general working speed correspondingly.

We present a tablet computer interaction study, during which different levels of $\mathrm{CW}$ are induced. The proposed experiment abstracts and emulates typical tasks employees have to fulfill in modern working environments. In total, 15 subjects participated in the experiment. To predict the $\mathrm{CW}$, we evaluated the heart rate (HR), the heart rate variability (HRV), the electrodermal activity (EDA) and the tablet computer's touch features (duration and pressure). A sparse feature subset was identified and tested by comparing the accuracy of multiple machine learners.

The work is structured as follows: In section 1 we introduce the theoretical background of $\mathrm{CW}$ and summarize related work. An overview of the used hardware and the conducted experiment is given in section 2. Furthermore, the applied machine learning methods are described. In section 3 the results of our feature selection and classification are shown. Subsequently, in section 4 follows a discussion of the results. Finally, we summarize our work in section 5 and give prospect on our future work. 


\subsection{BACKGROUND}

Up to now, there is no universal definition of mental or cognitive workload. (Cain, 2007) summarized mental workload as the capabilities and effort of the operators in the context of a specific situation. Hence, CW is not an univariate, but a "multifaceted" entity. A comprehensible definition states CW to be: "an allencompassing term that includes any variable reflecting the amount or difficulty of one's work" (Bowling and Kirkendall, 2012). We will follow that definition within this work.

The measurement of $\mathrm{CW}$ is as divergent as its definition. CW can either be measured subjectively (selfreported) via performance measures (primary or secondary task e.g. error rate, or time-on-task) or by utilizing psycho-physiological measures (Cain, 2007).

Psycho-physiological measures are thereby based on the physiological responses of the human body, resulting from a psychological strain (e.g. cognitive workload). These physiological responses are controlled by the autonomic nervous system, which consists of the sympathetic and parasympathetic nervous system. Both systems regulate body functions accordingly to environmental conditions (e.g. increase alertness in challenging situations). Well known measurements to quantify these body functions and therefore to predict $\mathrm{CW}$ are based on the heart rate (HR) or heart rate variability (HRV) (Jorna, 1992), as well as on the electrodermal activity (EDA; or galvanic skin response - GSR) (Isshiki and Yamamoto, 1994).

\subsection{RELATED WORK}

The possibility of predicting psychological strain has frequently been presented in recent research. Physiological strain is thereby often referred to as mental or cognitive workload or more generally as stress ${ }^{1}$.

For instance (Choi and Gutierrez-Osuna, 2009) demonstrated the effectiveness of heart rate monitors in detecting mental stress. They highlighted the importance of an unobtrusive design to obtain high user acceptance rates. With their approach they were able to distinguish between stressed and non-stressed mental states with an accuracy of $69 \%$.

Within the work of (Wijsman et al., 2011) and (Choi et al., 2012) it was shown that the combination of the heart rate and additional predictors (e.g. respiration rate and EDA) improves the prediction accuracy $(79 \%$ and $81 \%)$.

Most recent work also emphasizes the problem of detecting CW (or stress) by considering physical ac-

\footnotetext{
${ }^{1}$ In some applications, e.g. the automotive industry, related parameters like arousal or fatigue are considered.
}

tivity as an additional predictor. (Karthikeyan et al., 2013) used physical activity information in order to prevent it from becoming a confounding factor. Their approach resulted in a prediction with an accuracy of up to $92.4 \%$.

Additionally, (Sun et al., 2012) focused on short term signal processing, which enables the detection of short term stress events. They presented remarkable results with a classification accuracy of up to $95 \%$. Yet, the topic of fine-grained stress or CW prediction has gained limited attention and is not individually addressed. Nevertheless, (Healey and Picard, 2005) predicted the perceived stress of drivers in three distinct gradations with accuracy up to $97 \%$.

In this work we focus on both, short-term signal processing of multiple parameters and fine-grained prediction of $\mathrm{CW}$. Both are mandatory requirements in order to implement $\mathrm{CW}$ prediction into adaptive assistive technology.

\section{METHODS}

The following section starts with an overview of the used sensory equipment (subsection 2.1). Detailed explanation of the conducted experiment (subsection 2.2) and the definition of ground truth (subsection 2.3) is provided afterwards. Finally, we outline mandatory signal processing steps (subsection 2.4) and refer the feature selection (subsection 2.5) and machine learning methods used within this work (subsection 2.6).

\subsection{HARDWARE}

The hardware setup is based on the Google Nexus 10 tablet computer(Nexus 10, 2012), which has sufficient computing power for the desired task and allows an easy integration of the external sensors.

The EDA was captured by using the Mindfield eSense Skin Response system(eSense Skin Response, 2015), which is a portable solution designed for tablet computers and smartphones. Its microphone jack is connected to the tablet computer and the two finger (hook and loop) electrodes are placed around the subject's index- and middle finger.

The Mindfield system was compared to a Brainproducts EDA sensor connected to a appertaining QuickAmp Amplifier(QuickAmp, 2015) as a reference system. Although both systems produced different outputs in terms of absolute value, their signals showed close agreement (Pearson's $r>0.8$ ). Therefore, we used the mobile and inexpensive Mindfield system. 
The heart rate was captured by two redundant systems. Firstly, we used an ECG based Polar H6 heart rate sensor(Polar H6, 2012), which is attached to a chest strap. Secondly the photoplethysmogram (PPG) based Mio Alpha watch(MIO Alpha, 2013) was used, which is worn around the wrist. Both heart rate sensors communicate wirelessly with the tablet computer via Bluetooth Low Energy. Measurement readings from both devices were comparable (mean deviation $3.85 \%$ ). However, we noted that the Mio Alpha smooths the measured values. For this reason, we only use data obtained from the Polar module in the following.

\subsection{EXPERIMENT}

We conducted an experiment to induce varying levels of $\mathrm{CW}$ during the interaction with a tablet computer. In total, 15 subjects volunteered to participate in the experiment. Subjects were mainly male students (14 male, 1 female, mean age $25.9 \pm 2.1$ ). All subjects were aware about the design of the experiment and gave their informed consent.

The total experiment lasted approximately 20 to 25 minutes for each participant and was repeated after a short break. During the break, the sensors were reapplied to increase robustness in terms of repeatability concerning the various sensors' attachment. Each pass of the experiment was divided into five phases:

1. Relaxation video ( 2 minutes)

2. Memorize items ( 3 to 4 minutes)

3. Stroop test ( 3 to 4 minutes)

4. Recall items (4 to 5 minutes)

5. Memory and reaction test (3 to 4 minutes)

The experiment started with a resting phase in which a relaxation video was presented to the subject (phase 1, video duration $90 \mathrm{~s}$ ). This was done in order to prevent possible effects resulting from the excitement of the ongoing experiment.

Afterwards, a memory test was initiated (phase 2). During this phase, 12 items of learning content were provided to the subject. The learning content consisted of demographic and economic data of the United States (first pass) and the Czech Republic (second pass). For each item, the time to memorize the provided information was limited to $10 \mathrm{~s}$.

Before the memorized content had to be recalled (phase 4) by the subject a Stroop test was carried out (phase 3, (Stroop, 1935)). During the Stroop test the user had to touch the button with the color that is identical to the color of a shown text on the screen (figure 1). The background color, the number of possible

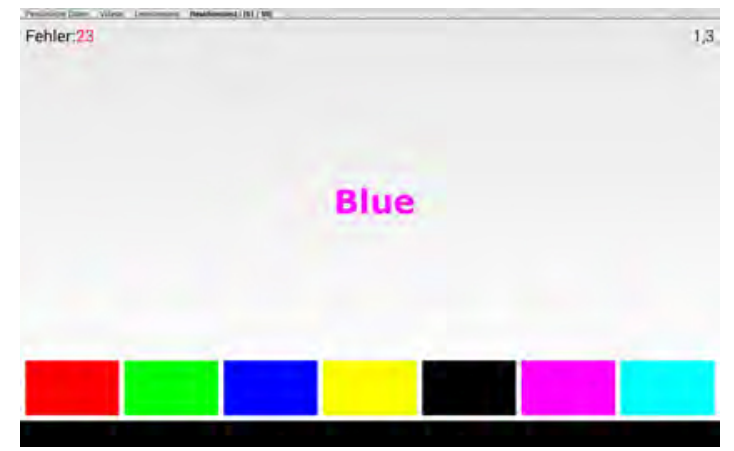

Figure 1: Example of the Stroop test in phase 3 of our tablet based experiment. Here, the user is asked to touch the magenta-colored button.

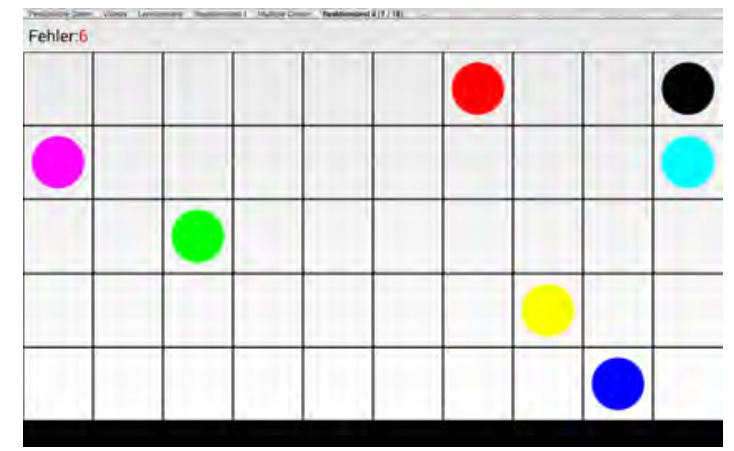

Figure 2: Checker board used to recall the color sequences in phase 5 of our tablet based experiment.

solutions (buttons) and the available time to answer was altered randomly. Hence, the Stroop test challenged the user with varying intensity levels. Overall, the subject was asked to reply to 90 Stroop items during 6 repetitions (15 items each). A short break preceded every repetition.

Afterwards, the subject was asked to recall the learning content from phase 2. This was done in a multiple-choice way, whereas 7 questions were composed into 3 blocks of varying difficulties. To increase the $\mathrm{CW}$ for the multiple-choice test in each block, the available time to answer was reduced $(7 \mathrm{~s}, 6 \mathrm{~s}$ and $5 \mathrm{~s}$, respectively). Additionally, in the last block, only invalid answers were provided.

Finally, the subject had to perform a mixed memory and reaction test (phase 5). For this test, colored circles were consecutively drawn on the screen. The subject's task was to memorize the color sequence and immediately recall it afterwards. The difficulty was altered by changing the count and duration of the circles shown ( 3 to 7 circle were shown for a duration of 700 to $500 \mathrm{~ms}$ each). Moreover, the number of used colors was changed randomly (3 to 7). To recall the color sequence, a checker board was presented to the subject (figure 2). The checker board was sparsely 
filled with colored circles (randomly distributed). The subject was asked to recall the color sequence, which was shown beforehand, by touching the corresponding circles.

The proposed experiment covers typical tasks workers are faced with in an abstract way. The abstraction focuses on the tasks to memorize and recall various working steps, e.g. while assembling a work piece or wiring a cable harness at the production line (mixed reaction and recall test, phase 5). The worker has to recall a new working process under time pressure. Another example is performing and following a diagnostic sequence. In this case the worker has to memorize facts and later on recall and compare the results (memory test: phase 2 and 4 ).

\subsection{GROUND TRUTH}

During the experiment, we simulated short-term stress events with varying intensities. Each event was assigned with an estimated or demanded $\mathrm{CW}$ by the experimenter. The annotation scale reached from 1 to 5. Yet, it is unclear if the subjects' perceived CW corresponds to the demanded $\mathrm{CW}$. Therefore, in order to obtain ground truth data, all participants were asked to self-report their perceived $\mathrm{CW}$ on a scale from 1 to 5. The self-report was enquired directly after a specific task was finished. Thereby, during each pass of the experiment, the subject was asked 17 times to give self-report of the perceived CW. This self-report was then assigned as ground truth (target label) for the previously performed task.

\subsection{PREPROCESSING AND FEATURE EXTRACTION}

The utilized Polar H6 provides the heart rate and the RR-interval for each recognized heart beat. Therefore, the data stream is recorded in non-uniform time intervals. To enable a common frequency based analysis the data is re-sampled to $4 \mathrm{~Hz}$ as suggested by (Singh et al., 2004). For the transformation into the frequency domain Welch's method in combination with a Hamming window is used. Prior to the feature extraction, the RR-interval is normalized and detrended as demonstrated by (Tarvainen et al., 2002). Furthermore, heart rate for each subject is min-max normalized to increase inter-subject comparability.

The EDA is captured with a sample rate of $10 \mathrm{~Hz}$. In order to remove outliers, we applied a low pass filter with a cut-off frequency of $0.5 \mathrm{~Hz}$. The raw EDA signal is decomposed into the skin conductance level (SCL) and skin conductance response (SCR), as described by (Choi et al., 2012). Their method is based on the approach from (Tarvainen et al., 2002), which was also used to detrend the RR-interval beforehand.

Statistical data (minimum, maximum, mean, standard deviation) is calculated from HR, RR-interval, EDA, SCR and SCL. In addition, amplitude, duration, area and frequency of the EDA and SCR signals are computed and commonly known features based on heart rate variability are used (Malik et al., 1996). As the experiment was carried out using a tablet computer, we additionally record mean pressure, mean duration and total count of touch events on the touch screen display during the experiment. A comprehensive overview of all extracted features is given in section 3.2.

Because the extracted features are not all commensurate, min-max scaling (equation 1) or ztransformation (equation 2) is used.

$$
\begin{gathered}
\operatorname{Min}-\operatorname{Max}(X)=\frac{X-\min (X)}{\max (X)-\min (X)} \\
\text { Z-Trans. }(X)=\frac{X-\bar{X}}{\sigma(X)} \\
\sigma(X)=\sqrt{\frac{1}{n-1} \sum_{i=1}^{n}\left(x_{i}-\bar{X}\right)^{2}} \text { and } \bar{X}=\frac{1}{n} \sum_{i=1}^{n} x_{i}
\end{gathered}
$$

\subsection{FEATURE SELECTION}

To identify the optimal window size and overlap, we derive multiple feature subsets based on the corresponding sensory element (HR, EDA, Touch). Then, we empirically explore the predictive performance for each combination of subset, window size and overlap. For this purpose, we refer to the mean accuracy from stratified 10-fold cross-validated Decision-Trees. Afterwards, we reduce the feature space to avoid redundancies. Therefore, all features are ranked by their information gain, utilizing Weka 3 data mining software (Witten and Frank, 2005).

\subsection{CLASSIFICATION}

With a comparative analysis we want to identify the potential of the derived feature set for the fine-grained and short-term prediction of CW. Therefore, we train multiple fine-grained supervised classification models with the optimal feature set and window size that was evaluated beforehand (section 3.2). We compare various well-known classifiers, using the correspondent MATLAB Toolbox(MATLAB, 2015) implementations. Evaluated methods are: Naïve Bayes, 
Decision-Tree, k-Nearest Neighbor and Support Vector Machine. Additionally, we set up a Gaussian Process Regression model utilizing the GMPML MATLAB Toolbox (Rasmussen and Nickisch, 2010).

The Naïve Bayes classifier provides a generative model of the feature space. It is used to estimate the probability distribution of the feature space given a specific class label. Thereby, the estimate is based on the (naïve) assumption that, given a certain class label, the corresponding predictors are conditionally independent to each other. (Webb, 2010)

The Decision-Tree classifier follows the devideand-conquer approach, meaning that multiple decision rules are created and arranged in a tree like structure. Thus, Decision-Trees allow non-parametric modeling, which at the same time, however, can lead to over-fitting. (Fürnkranz, 2010)

The k-Nearest Neighbor classifier belongs to the group of lazy or instance-based learners. The classification is based on querying the similarity (or distance) of a new observation to the known observations from the training set. Typically, a Euclidean distance measure is used. Each new observation is then classified by majority vote in respect to its k nearest neighbors. (Keogh, 2010)

The Support Vector Machine is a kernel-based discriminative classifier. Utilizing the kernel trick (Bishop, 2006) the Support Vector Machine constructs a hyperplane that allows non-linear separation of the feature space. Often polynomial, Gaussian or radial-basis functions are used as kernel functions. In order to enable multiclass classification with Support Vector Machine, we make use of the MATLAB ErrorCorrecting Output Codes implementation (Dietterich and Bakiri, 1995).

Lastly, we train a Gaussian Processes Regression (also known as Kriging), which is a non-parametric kernel-based model. In the Gaussian Process Regression, the observations of the training set are seen as random samples from a multivariate Gaussian distribution. The prediction is based on a Gaussian process, which is defined by a mean and a covariance function. To attain class labels, we round the output values of the regression. (Bishop, 2006)

To compare predictive performance, we refer to accuracy (equation 3), sensitivity (true positive rate, equation 4), specificity (true negative rate, equation 5) and precision (positive predictive value, equation 6). To prevent overfitting and assure validity of the classifier, we make use of stratified 10-fold crossvalidation.

$$
\text { Accuracy }=\frac{T P+T N}{T P+F P+T N+F N}
$$

$$
\begin{aligned}
& \text { Sensitivity }=\frac{T P}{T P+F N} \\
& \text { Specificity }=\frac{T N}{T N+F P} \\
& \text { Precision }=\frac{T P}{T P+F P} \\
& \text { TP - True Positive FP - False Positive } \\
& \text { TN - True Negative FN - False Negative }
\end{aligned}
$$

\section{RESULTS}

In this section, we present findings from the experiment (section 3.1) and reveal the selected feature subset (section 3.2). Finally, we compare results of the trained classifiers (section 3.3).

\subsection{EXPERIMENT}

To verify that the subjects were adequately challenged during the experiment we compare the demanded $\mathrm{CW}$ with the self-reported $\mathrm{CW}$ (ground truth). In direct comparison, the demanded $\mathrm{CW}$ level of the experiment mostly coincided with the subjects' selfreported $\mathrm{CW}$ level (figure 3). However, while the subjects were performing tasks with demanded CW level of 4 and 5 no significant difference between the self-reported CW levels were found (1-way ANOVA, $p=0.27)$. We conclude, that the subjects were equally challenged during both tasks. Furthermore, the tasks with a demanded CW level 3 were experienced equally or even less challenging than the tasks with a demanded CW level 2 by the majority of the subjects. This could be explained through the effect of habituation during the experiment. The demanded $\mathrm{CW}$ could have been overestimated by the subjects,

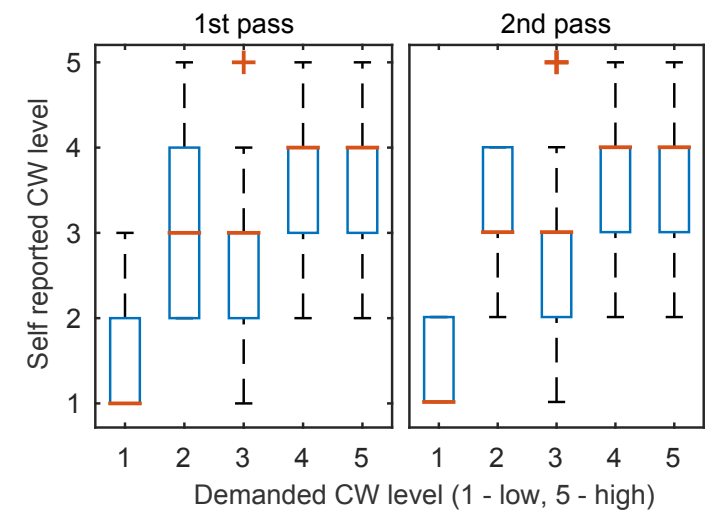

Figure 3: Distribution of the self-reported $\mathrm{CW}$ level during the 1 st and 2 nd pass of the experiment, grouped by the demanded CW level. 
thus the expectation may additionally confounded the self-reported CW (Harris et al., 1993). Nevertheless, the self-reported CW with 5 distinct levels is used, because 14 subjects (93\%) self-reported 4 or 5 different $\mathrm{CW}$ levels during the experiment. Only one subject reported CW with just three different levels (1 to 3).

Additionally, we verified repeatability of the experiment by comparing the first and the second pass of the experiment. We found similar mean and variance concerning the self-reported $\mathrm{CW}$ levels during the different experimental phases (figure 3). With paired t-test the null hypothesis that the self-reported $\mathrm{CW}$ between first and second pass are equal could not be rejected ( $p=0.14)$. Thus, we conclude there was no significant difference in the perceived stress level during both passes of the experiment.

\subsection{FEATURE SELECTION}

We extracted a total of 49 features (table 1) from the different sensor elements (HR, EDA, touch) ${ }^{2}$.

Table 1: Overview of all extracted features.

\begin{tabular}{ll}
\hline Source & Feature \\
\hline HR & mean, standard deviation, min., max. \\
\cline { 2 - 2 } RR & $\begin{array}{l}\text { mean, standard deviation, min., max., } \\
\text { pRR50, RMSSD, SD1, SD2, SD1/2, } \\
\text { skew, kurtosis, VLF, LF, nLF, nHF, } \\
\text { LF/HF }\end{array}$ \\
\hline SCL & mean, standard deviation, min., max., \\
\cline { 2 - 2 } & $\begin{array}{l}\text { mean, standard deviation, min., max., } \\
\text { peak count, peak prominence, max. } \\
\text { peak prominence, mean peak promi- } \\
\text { nence, median peak prominence, peak } \\
\text { duration, peak area }\end{array}$ \\
\hline Touch & mean duration, mean pressure, count \\
\hline
\end{tabular}

To determine an appropriate window size for the feature extraction, we defined multiple feature subsets. For every subset we extracted features and varied the length of the time window from 10 to $60 \mathrm{~s}$ in $5 \mathrm{~s}$ steps. We additionally altered the time window overlap. To generate overlapping windows the signal window is shifted by $25 \%, 50 \%, 75 \%$ or $100 \%$ (no overlap) of the length of the time window. To preestimate the usability and to determine the optimal window size and overlap, we evaluated the accuracy of 10-fold cross-validated Decision-Trees for each of the 308 possible feature sets (table 2).

\footnotetext{
${ }^{2}$ Detailed information can be found in table 5 located in the appendix.
}

Table 2: Best classification accuracy for each feature subset in respect to window size and overlap.

\begin{tabular}{lccc}
\hline Subset & Length & Overlap & Accuracy \\
\hline ALL & $35 \mathrm{~s}$ & $75 \%$ & $62.22 \%$ \\
\hline HR & $60 \mathrm{~s}$ & $75 \%$ & $51.54 \%$ \\
EDA & $30 \mathrm{~s}$ & $75 \%$ & $50.50 \%$ \\
TOUCH & $45 \mathrm{~s}$ & $75 \%$ & $46.01 \%$ \\
\hline HR \& EDA & $25 \mathrm{~s}$ & $75 \%$ & $60.16 \%$ \\
HR \& TOUCH & $50 \mathrm{~s}$ & $75 \%$ & $58.21 \%$ \\
TOUCH \& EDA & $35 \mathrm{~s}$ & $75 \%$ & $55.89 \%$ \\
\hline
\end{tabular}

For all tested combinations of window length, overlap, optimal accuracy for each feature subset was found with $75 \%$ overlap. With regard to the window length, the results were not equally consistent. Except for the heart rate feature set (Pearson's $r=0.9503$, $p<0.05)$, we found no significant trend or correlation between the classifier's performance and the length of the time windows. We conclude that there is no all-encompassing optimal window size or overlap, but each subset has its own optimum (figure 4).

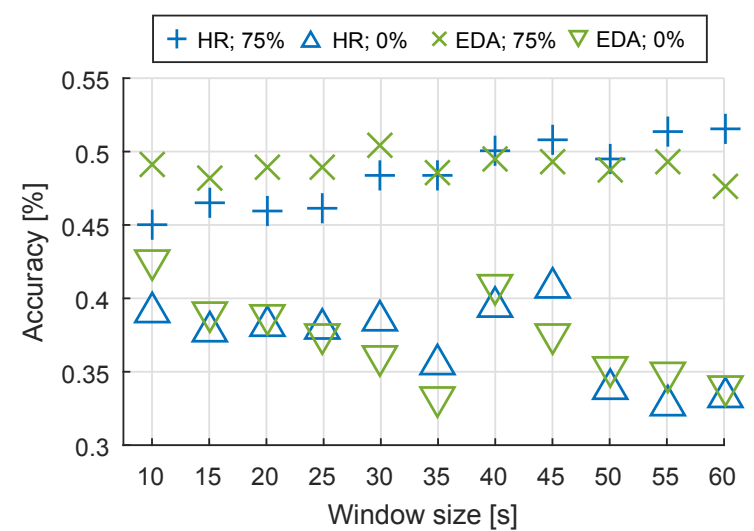

Figure 4: Mean accuracy from 10-fold cross-validated Decision-Tree trained on the heart rate and EDA feature subsets. Features were extracted on time windows with length of 10 to $60 \mathrm{~s}$ in $5 \mathrm{~s}$ steps. For the sake of clarity only $75 \%$ and $0 \%$ overlap are depicted.

Following the objective to set up a short-term prediction of $\mathrm{CW}$, the window size needs to be as short as possible. On the other hand, we need to keep a minimal length in order to obtain reliable features, e.g. from the heart rate sensor. We found that a $40 \mathrm{~s}$ window resulted in a good predictive performance concerning the heart rate features as well as the EDA features (figure 4). Hence, for further analysis, we chose a window size of $40 \mathrm{~s}$ with an overlap of $75 \%$. With this compromise, we fit with the classification accura- 
cies and keep the window size short at the same time. Nevertheless, due to the overlap, we obtain a new estimate every $10 \mathrm{~s}$. The chosen window length is by $20 \mathrm{~s}$ smaller compared to related work from (Sun et al., 2012) or (Karthikeyan et al., 2013).

Next, we select an optimal feature subset. From the first test, we found maximum accuracy by using the full feature set. However, to reduce interdependencies and redundancies within the full feature set we want to identify the most valuable features and deduce a sparse feature subset. Therefore, we ranked all features by their information gain (table 3 ).

Table 3: Average information gain and standard deviation for the top 12 ranked features. Selected features for the sparse feature subset are printed bold.

\begin{tabular}{lc}
\hline Feature & Information Gain \\
\hline Minimum EDA & $0.486 \pm 0.006$ \\
Average SCL & $0.451 \pm 0.004$ \\
Average EDA & $0.451 \pm 0.004$ \\
Maximum EDA & $0.416 \pm 0.003$ \\
Average touch duration & $0.361 \pm 0.004$ \\
Average touch pressure & $0.333 \pm 0.003$ \\
Minimum heart rate & $0.323 \pm 0.020$ \\
Maximum heart rate & $0.228 \pm 0.012$ \\
Average heart rate & $0.199 \pm 0.004$ \\
standard deviation SCR & $0.151 \pm 0.003$ \\
Average RR & $0.116 \pm 0.003$ \\
Maximum GSR peak prominence & $0.098 \pm 0.002$ \\
\hline
\end{tabular}

Using information gain, we found EDA features to be most important. Although the touch features showed worst predictive performance beforehand (Decision-Tree, table 2), they were ranked second most important after the EDA features. With further analysis, we have to assume that this result is due to spurious relationship within the experimental design. We must note that the expected count of touch events was not evenly distributed among the different phases of the experiment or the demanded CW levels. Furthermore, there was no control setting for touch pressure or duration, between the touch intensive and challenging phases (phase 3 and 5) and those phases that required only few or no touch inputs (phase 1, 2, 4). For this reason, we withdraw touch features from further analysis.

To reduce the total complexity of the feature space, the maximum prominence peak feature (derived from the EDA signal) was also withdrawn. The resulting sparse feature set contains the 9 most valuable features (regarding information gain), which include 5 features based on EDA and 4 heart rate based features (table 3 ).

\subsection{PREDICTIVE PERFORMANCE}

To evaluate the quality of the selected feature subset, we tested multiple classifiers and compared their accuracy (table 4).

Lowest accuracy resulted from Naïve Bayes classifier $(45.09 \pm 2.08 \%)$. We tested normal distributions as well as multiple kernel smoothing density estimates for the probability density. Regardless of the configuration, no perceptibly difference in the accuracy could be found. One explanation for the low accuracy is the lack of independence concerning the feature set. However, a thorough investigation of the cause is not part of this work.

For the Decision-Tree based classifier an average accuracy of $60.13 \pm 4.05 \%$ was achieved. In order to avoid over-fitting, we chose a limit of 100 splits per tree. Maximum average sensitivity is found on level $1(80.49 \pm 7.56 \%)$. However, the mean sensitivity considering levels 2,3 and 4 reached only $56.75 \pm 9.45 \%$. Thus, the misclassifications (or inaccuracy) mainly resulted from the confusions on the CW levels 2, 3 and 4. Comparable results are found with the classifier's specificity.

The usage of k-Nearest Neighbor resulted in an enhanced accuracy and overall sensitivity. Again, the highest sensitivity is found with level 1 $(82.14 \pm 5.35 \%)$. Compared to the Decision-Tree based classifier, the critical confusion on self-reported CW levels 2, 3 and 4 is reduced (sensitivity: $65.38 \pm 6.75 \%$ ). However, we noticed a continuous drop of the accuracy with a growing neighborhood. Best results were found with $k=1$, which could suggest an over-fitted model. For instance if the neighborhood is set to $k=10$, accuracy declines to $58.96 \pm 2.05 \%$.

Using Support Vector Machine we were able to further reduce confusion in the mid-levels (sensitivity: $69.10 \pm 7.74 \%$ ) and therefore increase the overall accuracy to $71.00 \pm 3.36 \%$. Best results were archived with radial-basis kernel, although usage of Gaussian or polynomial kernel did only slightly affect the predictive performance.

In consideration of the observed confusion in the mid-levels of the CW prediction, we infer both the target values (self-reported $\mathrm{CW}$ ) and the predictors (EDA, HR) to be noisy. Taking the assumption of noisy predictors and target values into account, we chose Gaussian Process Regression as an additional learner for the comparison. Gaussian Process Regression is well known to act as a linear smoother and therefore generally provide good predictive power in noisy settings (Quadrianto et al., 2010). Indeed, the Gaussian Process based classification outperformed 
Table 4: Comparison of different classifiers based on the sparse feature subset in descending order of 5-class accuracy. All tests are 10-fold cross validated. Standard deviation during the cross correlation is given with the mean accuracy.

\begin{tabular}{lllcc}
\hline Classifier & Normalization & Settings & Accuracy, 5-class & Accuracy, 2-class \\
Gaussian Process & min-max & Matérn kernel & $74.05 \pm 3.11 \%$ & $96.03 \pm 1.47 \%$ \\
Support Vector Machine & $\mathrm{z}$ & radial-basis kernel & $71.00 \pm 3.36 \%$ & $91.43 \pm 1.51 \%$ \\
k-Nearest Neighbor & $\mathrm{z}$ & euclidean distance & $67.90 \pm 3.98 \%$ & $92.10 \pm 2.34 \%$ \\
Decision-Tree & min-max & pruned; 100 split limit & $60.13 \pm 4.05 \%$ & $90.85 \pm 1.76 \%$ \\
Naïve Bayes & min-max & Gaussian kernel & $45.09 \pm 2.08 \%$ & $84.37 \pm 3.20 \%$ \\
\hline
\end{tabular}

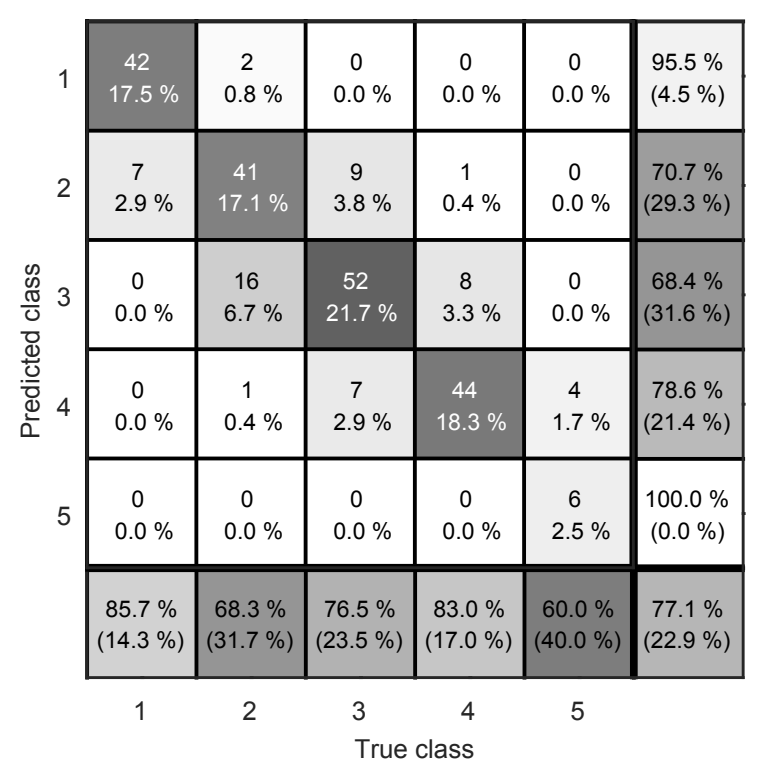

Figure 5: Confusion matrix for the best Gaussian Process based CW prediction (during 10-fold cross-validation). Last row contains sensitivity together with false negative rate (bracketed). The last column contains precision together with false discovery rate (bracketed).

the other methods with an accuracy of $74.05 \%$ (figure 5). Additionally, the mean sensitivity concerning predicted $\mathrm{CW}$ level 2, 3 and 4 was enhanced $(72.95 \pm 8.10 \%)$.

Still, not all uncertainties are covered by the Gaussian Process. This can easily be seen by shrinking the classification task to a binary problem. In this case self-reported CW level 1 is interpreted as no CW. All remaining levels are taken as present $\mathrm{CW}$. By reducing the machine learning task to this binary problem, the average accuracy for the Gaussian Process reaches up to $96.03 \pm 1.47 \%$. For the binary classification task, the ranking of the predictive power (accuracy) of the other tested classifiers remains mainly unchanged. In contrast to the fine-grained tasks, the Naïve Bayes classifier also provided an acceptable classification rate.

\section{DISCUSSION}

Within this work, we successfully demonstrated a fine-grained prediction of $\mathrm{CW}$. By focusing on a finegrained prediction based on short-term signals, we extended the complexity of the classification task. Additionally, we reached the accuracy of today's state of the art publications for the binary classification task. In comparison, the fine-grained classification resulted in a lower overall accuracy. This was explained by a low sensitivity regarding mid-level $\mathrm{CW}$ levels. This observed variation in the self-reported CW levels is partly explained due to the subjective perception of $\mathrm{CW}$. In future work the usage of more detailed selfreports (e.g. based on NASA- Task Load Index (Hart and Staveland, 1988)) could overcome this issue. Additionally, performance measures like error rate or time-on-task could further clarify the level of subjectively perceived CW. Nevertheless, regarding the Gaussian Process model, misclassification rarely exceeded more than one class (or level). Therefore, despite the lower overall accuracy, the fine-grained prediction should be favorable, because it facilitates a detailed specification of the perceived $\mathrm{CW}$.

Although Gaussian Process showed best accuracy, Support Vector Machine yielded comparable accuracy. As Support Vector Machines are more widespread and computationally efficient implementations are commonly available, they might be used preferentially.

Ranking of the extracted features revealed EDA features to contain maximum information content, directly followed by the heart rate features. As emphasized by (Sun et al., 2012) care has to be taken if heart rate is chosen as a predictor, because it is possibly influenced by means of physical activity. However, during our experiment the subjects were monitored by the experimenter, thus we can exclude physical activity as confounding factor. Nevertheless, the observed confounding influence of the touch features has to be considered in future tablet computer based experiments.

Yet, we found that even a narrow short-term feature subset is sufficient to precisely estimate a person's cognitive workload. This is a mandatory re- 
quirement in order to set up an adaptive assistive system, which is capable of balancing a given tasks' complexity accordingly to the users cognitive capacity.

\section{SUMMARY AND CONCLUSION}

We were able to achieve a fine-grained prediction of cognitive workload (stress), which exceeds the complexity of the ordinary binary classification task. Additionally, short-term feature were utilized. To reproduce a realistic setting, modern working environments were simulated in the presented experimental setup. The subjects self-reported their perceived $\mathrm{CW}$ directly after each task. After the preprocessing we were able to extract a total of 49 features. The most significant features and their ideal window size and overlap were determined with an initial estimate based on 10-fold cross-validated Decision-Trees. The identified sparse feature subset contains 9 features, which include 5 features based on EDA and 4 heart rate based features. The feature subset was then evaluated, by comparing the accuracy of multiple well established machine learning methods.

In conclusion, we achieved a classification accuracy of $96.03 \%$ for the binary $\mathrm{CW}$ prediction task and an accuracy of $74.05 \%$ for the fine-grained predictive model. This is likely to enable the development of more advanced assistive technology that can precisely adjust to the user's requirement in modern working environments.

In future work we plan to integrate the utilized sensors into a wearable and hands-free system. This will allow field studies in real working environments including skilled manual work. Additionally, the usage of more detailed self-reports is planned. Furthermore, we want to investigate how our fine-grained prediction of $\mathrm{CW}$ can be used to adapt the complexity of a task to the user's needs.

\section{ACKNOWLEDGEMENTS}

This research was supported by the DFG CoE 277: Cognitive Interaction Technology (CITEC), the German Federal Ministry of Education and Research (BMBF) within the Leading-Edge Cluster "Intelligent Technical Systems OstWestfalenLippe" (it's OWL), managed by the Project Management Agency Karlsruhe (PTKA), the BMBF project ALUBAR, and the $\mathrm{PhD}$ program "Design of Flexible Work Environments - Human-Centric Use of Cyber-Physical Sys- tems in Industry 4.0" supported by the North RhineWestphalian funding scheme "Fortschrittskolleg".

The authors are responsible for the contents of this publication.

The authors would like to thank Mindfield for providing the API for their eSense Skin Response system.

\section{REFERENCES}

Bishop, C. M. (2006). Pattern Recognition and Machine Learning (Information Science and Statistics), chapter Kernel Methods, pages 291-323. Springer New York.

Botthof, A. and Hartmann, E. (2015). Zukunft der Arbeit in Industrie 4.0 - Neue Perspektiven und offene Fragen. In Botthof, A. and Hartmann, E. A., editors, Zukunft der Arbeit in Industrie 4.0, pages 161-163. Springer Berlin Heidelberg.

Bowling, N. A. and Kirkendall, C. (2012). Workload: A Review of Causes, Consequences, and Potential Interventions, pages 221-238. John Wiley \& Sons, Ltd.

Cain, B. (2007). A review of the mental workload literature. In RTO-TR-HFM-121-Part-II. NATO Science and Technology Organization.

Choi, J., Ahmed, B., and Gutierrez-Osuna, R. (2012). Development and evaluation of an ambulatory stress monitor based on wearable sensors. IEEE Transactions on Information Technology in Biomedicine, 16(2):279-286.

Choi, J. and Gutierrez-Osuna, R. (2009). Using heart rate monitors to detect mental stress. In Sixth International Workshop on Wearable and Implantable Body Sensor Networks, pages 219-223.

Dietterich, T. G. and Bakiri, G. (1995). Solving multiclass learning problems via error-correcting output codes. Journal of artificial intelligence research, pages 263 286.

eSense Skin Response (2015). Biofeedback system. Mindfield Biosystems Ltd., Berlin, Germany.

Fürnkranz, J. (2010). Decision tree. In Sammut, C. and Webb, G., editors, Encyclopedia of Machine Learning, pages 263-267. Springer US.

Harris, W., Hancock, P., and Arthur, E. (1993). The effect of taskload projection on automation use, performance, and workload. In Proceedings of the Seventh International Symposium on Aviation Psychology.

Hart, S. G. and Staveland, L. E. (1988). Development of NASA-TLX (task load index): Results of empirical and theoretical research. In Human Mental Workload, volume 52, pages 139-183. North-Holland.

Healey, J. and Picard, R. (2005). Detecting stress during real-world driving tasks using physiological sensors. IEEE Transactions on Intelligent Transportation Systems, 6(2):156-166.

Isshiki, H. and Yamamoto, Y. (1994). Instrument for monitoring arousal level using electrodermal activity. In 
Proceedings of IEEE International Conference on Instrumentation and Measurement Technology, pages 975-978. IEEE.

Jorna, P. G. (1992). Spectral analysis of heart rate and psychological state: a review of its validity as a workload index. Biological psychology, 34(2-3):237-257.

Karthikeyan, P., Murugappan, M., and Yaacob, S. (2013). Detection of human stress using short-term ECG and HRV signals. Journal of Mechanics in Medicine and Biology, 13(02): 1350038 .

Keogh, E. (2010). Nearest neighbor. In Sammut, C. and Webb, G., editors, Encyclopedia of Machine Learning, pages 714-715. Springer US.

Malik, M., Bigger, J. T., Camm, A. J., Kleiger, R. E., Malliani, A., Moss, A. J., and Schwartz, P. J. (1996). Heart rate variability. European Heart Journal, 17(3):354-381.

MATLAB (2015). Version 8.5.0 (R2015a). The MathWorks Inc., Natick, Massachusetts.

MIO Alpha (2013). Heart rate watch. Physical Enterprises Inc. (Mio Global), Canada, Vancouver.

Nexus 10 (2012). GT-P8110. Google Inc.; Samsung Electronics.

Polar H6 (2012). Model: X9. Polar Electro Oy, Finland, Kempele.

Quadrianto, N., Kersting, K., and Xu, Z. (2010). Gaussian process. In Sammut, C. and Webb, G., editors, Encyclopedia of Machine Learning, pages 428-439. Springer US.

QuickAmp (2015). Biofeedbacksystem. Brain Products $\mathrm{GmbH}$, Gilching, Germany.

Rasmussen, C. E. and Nickisch, H. (2010). Gaussian processes for machine learning (GPML) toolbox. Journal of Machine Learning Research, 11:3011-3015.

Rouse, W., Edwards, S., and Hammer, J. M. (1993). Modeling the dynamics of mental workload and human performance in complex systems. IEEE Transactions on Systems, Man, and Cybernetics, 23(6):1662-1671.

Singh, D., Vinod, K., and Saxena, S. (2004). Sampling frequency of the RR interval time series for spectral analysis of heart rate variability. Journal of medical engineering \& technology, 28(6):263-272.

Stroop, J. R. (1935). Studies of interference in serial verbal reactions. Journal of Experimental Psychology, 18(6):643-662.

Sun, F.-T., Kuo, C., Cheng, H.-T., Buthpitiya, S., Collins, P., and Griss, M. (2012). Activity-aware mental stress detection using physiological sensors. In Gris, M. and Yang, G., editors, Mobile Computing, Applications, and Services, volume 76, pages 211-230. Springer Berlin Heidelberg.

Tarvainen, M., Ranta-aho, P., and Karjalainen, P. (2002). An advanced detrending method with application to HRV analysis. IEEE Transactions on Biomedical Engineering, 49(2):172-175.

Wallhoff, F., Ablassmeier, M., Bannat, A., Buchta, S., Rauschert, A., Rigoll, G., and Wiesbeck, M. (2007). Adaptive human-machine interfaces in cognitive production environments. In Proceedings of IEEE Inter- national Conference on Multimedia and Expo, pages 2246-2249.

Webb, G. (2010). Naïve bayes. In Sammut, C. and Webb, G., editors, Encyclopedia of Machine Learning, pages 713-714. Springer US.

Wijsman, J., Grundlehner, B., Liu, H., Hermens, H., and Penders, J. (2011). Towards mental stress detection using wearable physiological sensors. In Proceedings of IEEE Engineering in Medicine and Biology Society, pages 1798-1801.

Witten, I. H. and Frank, E. (2005). Data Mining: Practical Machine Learning Tools and Techniques. Morgan Kaufmann Publishers Inc., San Francisco, CA, USA, second edition.

Young, M. S. and Stanton, N. A. (2002). Attention and automation: New perspectives on mental underload and performance. Theoretical Issues in Ergonomics Science, 3(2):178-194.

\section{APPENDIX}

Table 5: Selected methods used for feature calculation.

\begin{tabular}{|c|c|c|}
\hline categorie & function & definition \\
\hline \multirow[t]{2}{*}{ time } & mean & $\mu=\frac{1}{n} \sum_{i=1}^{n} x_{i}$ \\
\hline & $\begin{array}{l}\text { standard } \\
\text { deviation }\end{array}$ & $\sigma=\sqrt{\frac{1}{n-1} \sum_{i=1}^{n}\left(x_{i}-\mu\right)^{2}}$ \\
\hline statistical & $\begin{array}{l}\text { skew } \\
\text { kurtosis }\end{array}$ & $\begin{array}{l}1 / n \sum_{i=1}^{n}\left(x_{i}-\mu\right)^{3} \\
1 / n \sum_{i=1}^{n}\left(x_{i}-\mu\right)^{4}\end{array}$ \\
\hline \multirow{6}{*}{$\begin{array}{l}\text { heart rate } \\
\text { variability }\end{array}$} & NN50 & $\sum_{i=1}^{n-1}\left(x_{i}-x_{i+1}>.05\right)$ \\
\hline & RMSSD & $\sqrt{1 / n \sum_{i=1}^{n}\left(x_{i}-x_{i+1}\right)^{2}}$ \\
\hline & SDSD & $\sigma\left(\left(x_{1}-x_{2}\right) \ldots\left(x_{n-1}-x_{n}\right)\right)$ \\
\hline & SD1 & $\sqrt{.5 \cdot S D S D^{2}}$ \\
\hline & SD2 & $\sqrt{\left(2 \cdot S D S D^{2}\right)-\left(.5 \cdot \sigma^{2}(x)\right)}$ \\
\hline & SD12 & $S D 1 / S D 2$ \\
\hline \multirow[t]{6}{*}{ (spectral) } & VLF & energy 0.00 to $0.04 \mathrm{~Hz}$ \\
\hline & LF & energy 0.04 to $0.15 \mathrm{~Hz}$ \\
\hline & $\mathrm{HF}$ & energy 0.15 to $0.40 \mathrm{~Hz}$ \\
\hline & $\mathrm{nLF}$ & normalized energy $(L F / L F+H F)$ \\
\hline & $\mathrm{nHF}$ & normalized energy $(H F / L F+H F)$ \\
\hline & $\mathrm{LF} / \mathrm{HF}$ & $L F / H F$ \\
\hline \multirow{4}{*}{$\begin{array}{l}\text { geometric } \\
\text { (peak) }\end{array}$} & count & number of peaks \\
\hline & prominence & $\begin{array}{l}\text { distance between to successive } \\
\text { peaks }\end{array}$ \\
\hline & width & $\begin{array}{l}\text { distance between the two mini- } \\
\text { mums surrounding a peak }\end{array}$ \\
\hline & area & $\begin{array}{l}\text { integral between the two mini- } \\
\text { mums surrounding a peak }\end{array}$ \\
\hline
\end{tabular}

